\title{
IDENTIFICATION OF METABOLITES OF SULFOBROMOPHTHALEIN *
}

\author{
By G. M. GRODSKY, J. V. CARBONE ANd R. FANSKA \\ (From the Gastrointestinal Clinic, San Francisco General Hospital [University of California \\ Service], the Metabolic Unit for Research in Arthritis and Allied Diseases, \\ and the Department of Medicine, University of California School \\ of Medicine, San Francisco, Calif.)
}

(Submitted for publication June 8, 1959; accepted July 23, 1959)

Sulfobromophthalein (BSP) is normally cleared from the blood by the liver and excreted into the bile. Brauer, Krebs and Pessotti (1) originally reported that part of the pigment appearing in dog bile was chromatographically different from the injected dye. Our studies using different chromatographic techniques indicate that in man the bulk of the recovered pigments (65 to 75 per cent, according to estimates based on their absorption at $575 \mathrm{~m} \mu$ ) consists of two metabolites. Similar metabolites are normally found in the urine and trace amounts also appear in the serum. The absorption spectra in the visible range and the indicator characteristics of BSP and the metabolic products were indistinguishable (2).

The liver apparently is capable of producing the metabolites, since they have been demonstrated in bile from preparations of isolated rat liver perfused with $\operatorname{BSP}(3,4)$. Variations in the concentration of BSP metabolites in serum and urine of patients with liver disease are reported elsewhere (5).

Since the chemical difference between the free dye and its metabolites elucidates in part the mechanism of hepatic function involved in BSP clearance, characterization of this structural difference has received considerable attention. BSP is a derivative of phenolphthalein, therefore both compounds could be excreted by the same pathway, that is, by conjugation with glucuronic acid (6). This mechanism for BSP clearance appears unlikely since the metabolites were stable to hydrolysis with $\beta$-glucuronidase $(4,7)$, with dilute acid and with dilute base, and gave a negative Dische reaction for uronic acid (4). Furthermore, both of the major metabolites in human bile were associated with ninhydrin-positive substances,

\footnotetext{
* Supported by a grant (A-2455) from the National Institutes of Health, United States Public Health Service, Bethesda, Md.
}

whose identification was complicated by the presence of high concentrations of ninhydrin-positive impurities arising from the bile itself (3). Several recent reports have also indicated that the BSP metabolites contain a ninhydrin-positive moiety (8-10). The present study reports the presence of glycine and glutamic acid as possible contaminants of the BSP metabolites and indicates that BSP is excreted as a mercaptide with cysteine or the peptide glutathione.

\section{METHODS AND MATERIALS}

E.xperimental. Human subjects with draining bile fistulas were injected with $5 \mathrm{mg}$. of BSP per $\mathrm{Kg}$. of body weight. Control specimens of bile were taken immediately before the dye was injected. Test specimens, in equal volume, were collected during a 30 to 60 minute interval after the injection.

Isolation of the crude metabolites. In initial experiments, the metabolites were isolated as follows. The total BSP pigments were extracted from bile with three volumes of acetone. The acetone extract was taken to dryness in z'acuo, then dissolved in water (approximately $0.2 \mathrm{ml}$. per ml. of initial bile). The solution was chromatographed on Whatman $3 \mathrm{MM}$ paper in an ascending system employing tert-butanol-water $(1.73: 1 \mathrm{v} / \mathrm{v})$ for six to 15 hours. The BSP bands were developed with ammonia fumes (or if permanent records were desired, by dipping the paper in a fresh saturated solution of sodium hydroxide in 90 per cent ethanol). After the position of the metabolites had been determined with ammonia, the bands were cut out and individually eluted with water. The resultant solutions were then taken to dryness.

Purification of the metabolites. Purified pigments were prepared by chromatography in three systems. Activated alumina (Alcoa, 80 to 200 mesh) was washed 10 times in two volumes of water and once in acetone. During each washing period, the larger particles were allowed to settle for one minute; the supernatant was then decanted and discarded. The dried alumina was suspended in a loading solution consisting of two volumes of acetone to one volume of water. Columns, $3.4 \mathrm{~cm}$. (outer diameter) by $6 \mathrm{~cm}$., were prepared by gravity packing. The dried acetone extract from $60 \mathrm{ml}$. of bile was sus- 


\section{BSP METABOLITES IN HUMAN BILE}

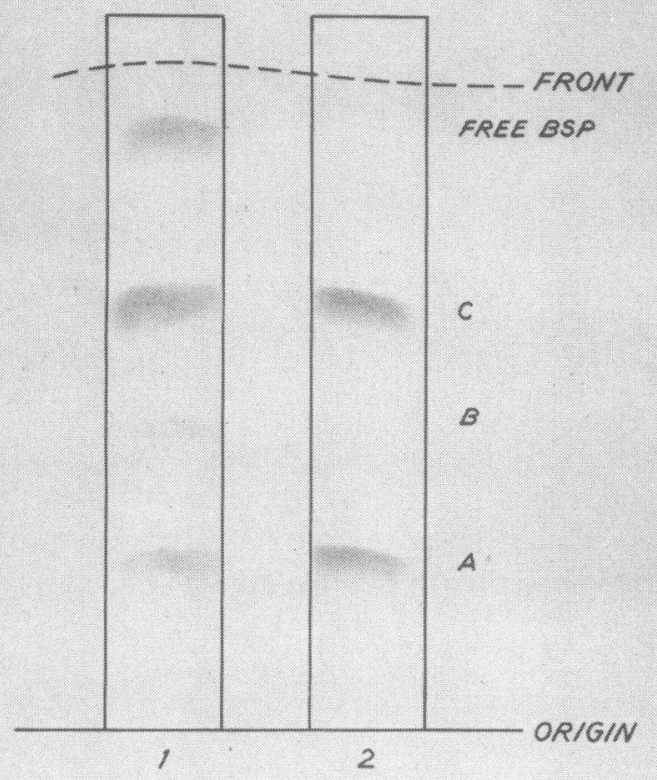

Fig. 1. Paper Chromatograph of BSP Pigments in Himan Bile (Drawing)

Ascending system employing tcrt-butanol-water was used. 1. BSP color developed with $\mathrm{NaOH} ; 2$. ninhydrin color developed with 0.1 per cent ninhydrin in ethanol.

pended in $45 \mathrm{ml}$. of loading solution and passed through the column. The column was then washed with an additional $200 \mathrm{ml}$. of the pure loading solution. Unconjugated BSP, amino acids and bile pigments were eluted with $600 \mathrm{ml}$. of a solution of concentrated ammonium hydroxide, water and acetone $(4: 46: 50 \mathrm{v} / \mathrm{v})$. The conjugated BSP remaining on the column was eluted with $125 \mathrm{ml}$. of acetone-water $(1: 9 \mathrm{v} / \mathrm{v})$. The eluate was taken to dryness, suspended in a minimal amount of water and subjected to ascending paper chromatography on Whatman 3 MM paper ${ }^{1}$ in a solvent system consisting of $n$-butanolwater $(1.48: 1 \mathrm{v} / \mathrm{v})$ made to a single phase solution by the addition of 0.27 volume of acetic acid. This system further removed traces of ninhydrin-positive impurities. After elution with water, the metabolites were separated by chromatography in the tert-butanol-water system used originally. The individual metabolites were then eluted with water, taken to dryness and stored in vacuo.

The equal volume of control bile obtained from each subject immediately before injection of BSP was treated similarly. Fractions corresponding in mobilities to the

1 To reduce ninhydrin-positive impurities, the paper was first washed 10 times with glass-distilled water. individual metabolites were isolated by employing the three chromatographic systems just described. The chemical characteristics of the control fractions were compared with those of the pigment fractions obtained from the bile containing BSP.

Hydrolysis of the metabolites. In initial experiments the individual metabolites were hydrolyzed in $6 \mathrm{~N}$ hydrochloric acid in a sealed glass tube at $120^{\circ} \mathrm{C}$. for 18 hours. After being repeatedly dried and redissolved in water to drive off traces of remaining hydrochloric acid, the hydrolysate was subjected to chromatography in the tertbutanol-water solvent system. The individual amino acids were subsequently identified by two-dimensional chromatography (11). Since hydrolysis with hydrochloric acid destroys several amino acids, including cysteine (12), this method was subsequently discarded in favor of hydrolysis with 48 per cent hydrobromic acid in a sealed tube at $120^{\circ} \mathrm{C}$. for 18 hours.

Hydrogenolysis with Raney-nickel. Raney-nickel catalyst W-2 was prepared as described by Mozingo (13). A sample of metabolite containing approximately 0.4 $\mathrm{mg}$. of BSP (by weight) was dissolved in $0.2 \mathrm{ml}$. of water and mixed with $200 \mathrm{mg}$. of Raney-nickel suspended in $2.5 \mathrm{ml}$. of ethanol. After being refluxed for two hours, the mixture was centrifuged and the supernatant was removed. The precipitate was washed two times with duplicate washes of $0.5 \mathrm{ml}$. of 50 per cent ethanol and five times with $0.5 \mathrm{ml}$. of 10 per cent ammonium hydroxide. The supernatant and washes were combined and taken to dryness in racuo. "Desulfurated glutathione" was prepared by substituting glutathione for the sample of the metabolite in the above procedure.

Synthesis of BSP-amino acid complexes. A solution consisting of $0.1 \mathrm{mMole}$ of free $\mathrm{BSP}$ and $0.1 \mathrm{mMole}$ of cysteine, adjusted to $2 \mathrm{ml}$. with water, was heated in a sealed tube at $120^{\circ} \mathrm{C}$. for 18 hours. The reaction mixture was taken to dryness and free BSP and the unreacted amino acids were removed by the same three chromatographic systems used for the BSP metabolites. The complexes, if any, were eluted with water and taken to dryness in r'acuo. Complex formation of BSP and the following amino acids or peptides was similarly attempted: cystine, glutathione, tryptophan, tyrosine, phenylalanine, glycine, glutamic acid, valine, alanine, serine. threonine, methionine, lysine hydrochloride and leucine.

\section{RESULTS}

The BSP metabolites obtained from bile reacted positively with ninhydrin (Figure 1). The possibility that the conjugates were complexes with protein is unlikely, since most of the proteins were either precipitated during the initial acetone extraction of bile or held at the origin in the chromatographic systems employed.

When BSP-A and BSP-C were eluted from the initial paper chromatograms and hydrolyzed in hydrochloric acid, ninhydrin-positive substances 
TABLE I

Release of ninhydrin-positive material from BSP metabolites after hydrolysis with $\mathrm{HCl}$

\begin{tabular}{|c|c|c|c|c|c|c|}
\hline & \multicolumn{3}{|c|}{ Before $\mathrm{HCl}$} & \multicolumn{3}{|c|}{ After $\mathrm{HCl}$} \\
\hline & Control & Metabolite & $\begin{array}{l}\text { Metabolite } \\
\text { minus } \\
\text { control }\end{array}$ & Control & Metabolite & $\begin{array}{c}\text { Metabolite } \\
\text { minus } \\
\text { control }\end{array}$ \\
\hline & \multicolumn{3}{|c|}{$\mu g . N^{*} / m l$ of fraction } & \multicolumn{3}{|c|}{$\mu g . N^{*} / m l$ of fraction } \\
\hline BSP-A & 1.1 & 8.1 & 7.0 & 19.9 & 30.2 & 10.3 \\
\hline BSP-A & 3.0 & 15.0 & 12.0 & 17.2 & 31.0 & 16.0 \\
\hline BSP-A & 2.4 & 12.6 & 10.2 & 12.6 & 25.7 & 13.1 \\
\hline BSP-C & 2.0 & 7.1 & 5.1 & 10.2 & 15.3 & 5.1 \\
\hline
\end{tabular}

* Quantitative ninhydrin determined by method of Moore and Stein (14). Glycine was used as the standard for calculating $\mu \mathrm{g}$. N.

were released, which when analyzed by twodimensional chromatography proved to be primarily glycine and glutamic acid. However, when samples of normal bile (which did not contain BSP) were chromatographed and fractions corresponding in mobility to the metabolites were eluted and hydrolyzed, large quantities of glycine and glutamic acid were again found. The amounts of ninhydrin-positive material released from the crude BSP fractions and from identical fractions of control bile before and after hydrolysis with hydrochloric acid are compared in Table I. (No attempt was made to establish the ratio of $\mathrm{BSP}$ to amino nitrogen in this crude mixture.) The content of ninhydrin-positive material in the BSP metabolites, which was higher than that of the control fractions before hydrolysis, was increased two- to fivefold after hydrolysis. Analysis of the controls, however, showed that most of this amino nitrogen came from the bile or paper (as glycine and glutamic acid) rather than from the BSP metabolites. The metabolites required further purification before their ninhydrin-positive constituents could be identified.

The chromatographic paper used in most of our studies contained considerable amounts of glycine and glutamic acid which were demonstrable only after acid hydrolysis. These impurities were substantially reduced by the described washing procedure. The electrophoretic mobility of bile-acetone extracts containing $\mathrm{BSP}$ were extensively studied with three systems using $0.25 \mathrm{M}$ acetic acid as the electrolyte: Durrum paper electrophoresis (15), hanging curtain electrophoresis (16) and starch block electrophoresis (17). The patterns obtained with the three systems were essentially the same. Unconjugated BSP moved rapidly toward the anode; the ninhydrin-positive metabolites moved less rapidly toward the anode and were usually indistinguishable from each other; large quantities of ninhydrin-positive impurities remained at the origin or moved toward the cathode. For large-scale preparations, electrophoresis was eventually abandoned in favor of chromatography on alumina columns, followed by chromatography in the two systems, butanol-water and $n$-butanol-water-acetic acid. On three occasions, the molar ratio of $\mathrm{BSP}$ to ninhydrin-positive amino nitrogen (with cysteine as a standard) of each metabolite ranged from 0.8 to 1.2. Control bile purified by this procedure was ninhydrinnegative before hydrolysis. Figure $2 \mathrm{~A}$ shows the results obtained after hydrolysis of the purified metabolites with hydrochloric acid. Glycine and glutamic acid were still found in the controls, as well as in the fractions containing the metabolites. When the concentrations of purified pigment were greater than $0.25 \mathrm{mg}$. per square $\mathrm{cm}$., a faint ninhydrin-positive streak corresponding to cysteine was usually detectable in the BSP fractions but not in the controls. The often reported (12) destruction of cysteine by hydrochloric acid was demonstrated when glutathione, a peptide containing glutamic acid, glycine and cysteine in equal quantities, was hydrolyzed in hydrochloric acid and chromatographed (Figure 2A). At low concentrations so much of the cysteine was destroyed that only glycine and glutamic acid were detectable.

\section{Hydrolysis with hydrobromic acid}

Some amino acids are comparatively stable during hydrolysis with hydrobromic acid (18). Furthermore, this reagent can be more effective than 
hydrochloric acid in hydrolyzing mercaptides (19). from both BSP purified metabolites (Figure 2B). In the chromatographic patterns obtained after The presence of cysteine was the only qualitative hydrobromic acid hydrolysis, cysteine was de- difference between the metabolites and the contectable as a discrete well-defined spot arising trols.

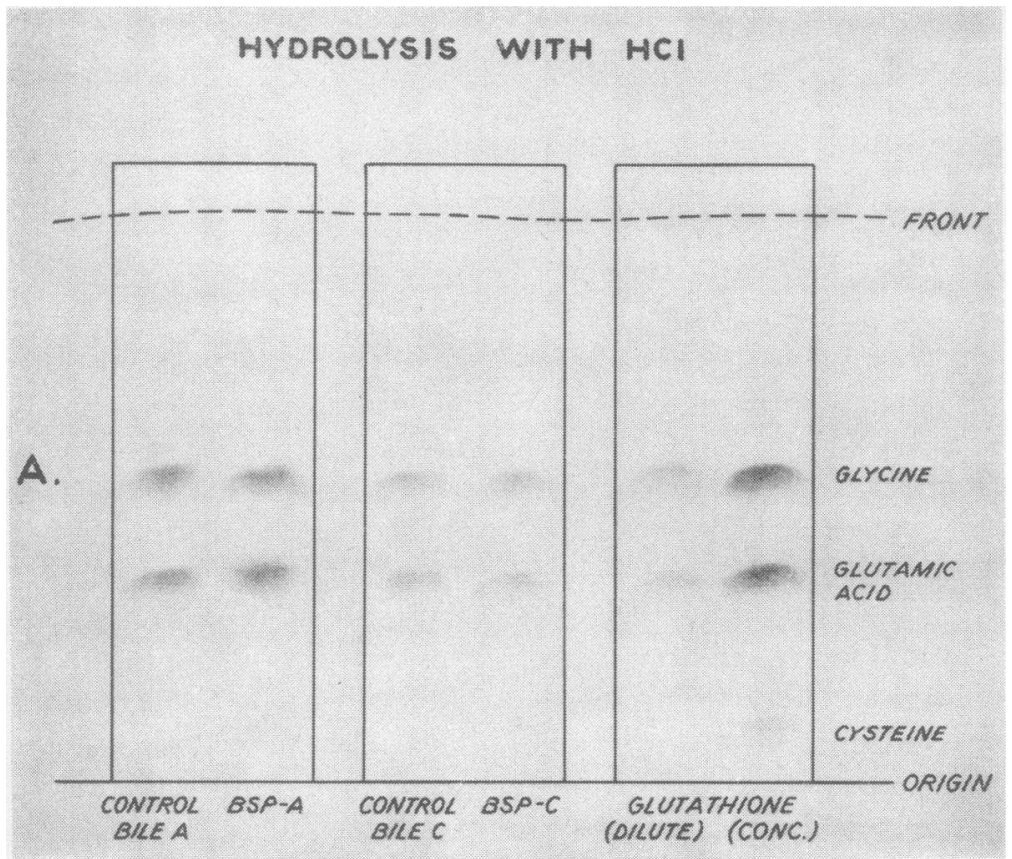

HYDROLYSIS WITH HBr

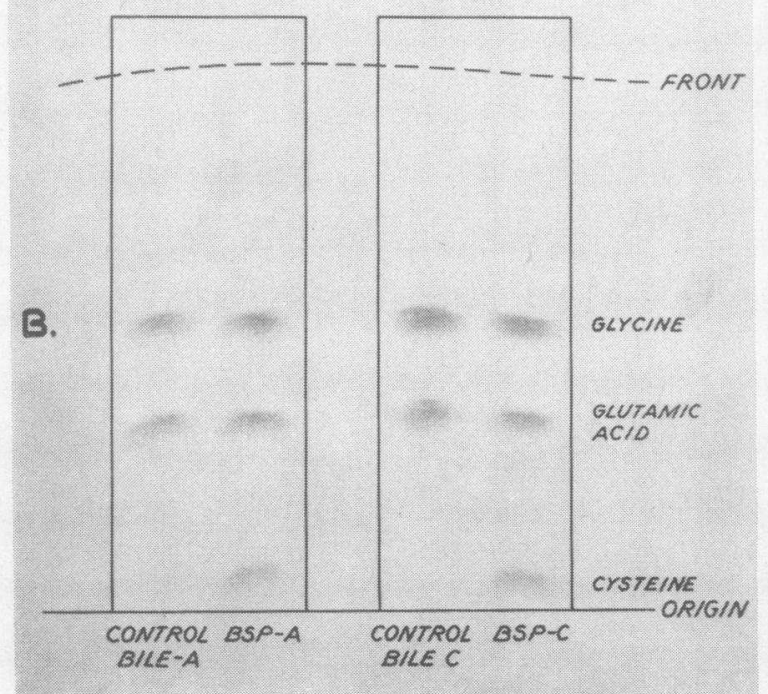

Fig. 2. Chromatography of Ninhydrin-Positive Substances Arising From Bile AND BSP Metabolites

Ascending system employing tert-butanol-water was used. A. Hydrolysis in $6 \mathrm{~N} \mathrm{HCl}$ at $120^{\circ} \mathrm{C}$.; B. hydrolysis with 48 per cent $\mathrm{HBr}$ at $120^{\circ} \mathrm{C}$.; C. hydrogenolysis with Raney-nickel. 


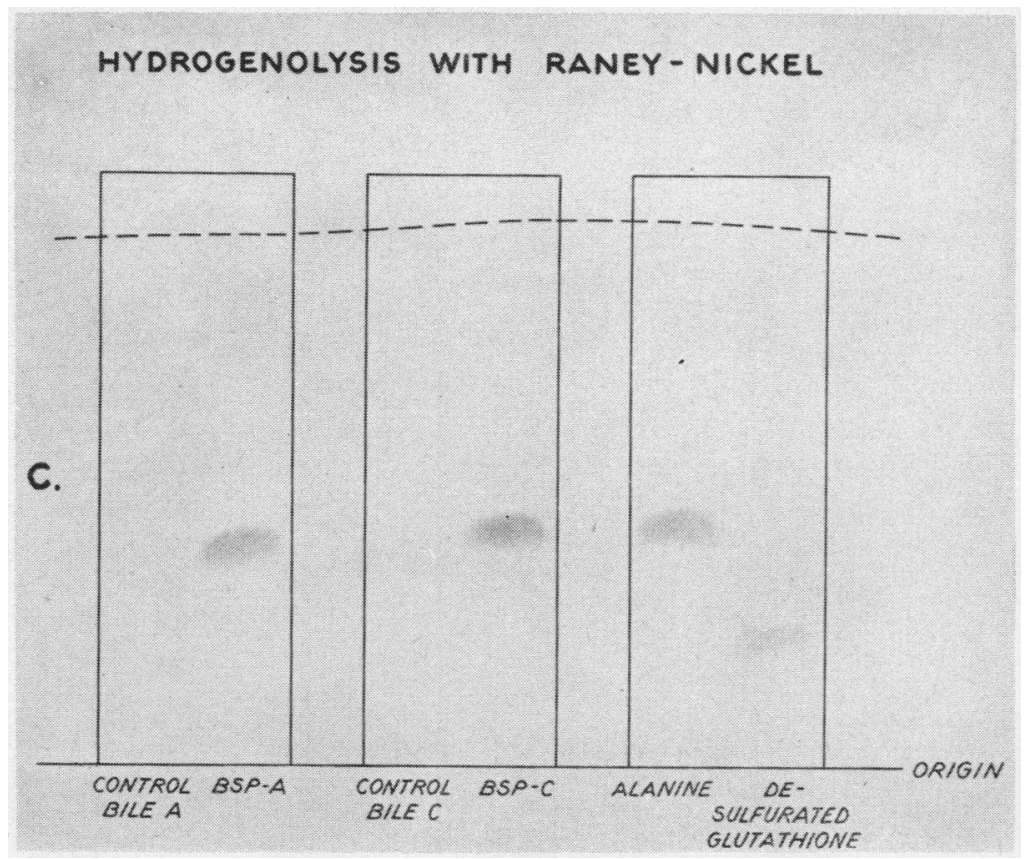

FIG. 2.-Continued

\section{Raney-nickel hydrogenolysis}

Hydrogenolysis with Raney-nickel breaks the carbon-sulfur bond in mercaptide linkages (20). If the BSP metabolites are conjugated with cysteine through the cysteine-sulfur bond, Raneynickel treatment should result in the degradation of the cysteine and the release of desulfurated cysteine (alanine). Furthermore, since this treatment does not hydrolyze peptide bonds, the interference of amino acids arising in the controls after hydrolysis with hydrochloric acid or hydrobromic acid is eliminated. When either purified BSP-A or BSP-C was treated with Raney-nickel and the resultant product was chromatographed (Figure 2C), only one major ninhydrin-positive spot was detected. This material was chromatographically identical to alanine after two-dimensional chromatography. There was no spot corresponding to desulfurated glutathione (the assumed product of BSP-glutathione degradation by Raney-nickel). No significant amount of ninhydrin-positive material was demonstrable in the controls after treatment with Raney-nickel.

\section{Staining with dichromate-silver nitrate}

Knight and Young reported that mercaptides and compounds with an available sulfhydryl group, when stained with potassium dichromate-silver nitrate, give a positive reaction characterized by an orange spot on a dark brown background (21). Both BSP-A and BSP-C reacted positively, while similar fractions from control bile were negative.

\section{Synthesis of BSP-amino acid complexes}

When BSP was incubated at $120^{\circ} \mathrm{C}$. with 14 different amino acids, only cysteine (or cystine) produced a complex with the pigment. ${ }^{2}$ After elimination of unreacted $\mathrm{BSP}$ and cysteine on the alumina column, the product contained BSP and cysteine in a molar ratio of 1 to 1 . When chromatographed in tert-butanol-water, the BSPcysteine complex moved as two spots, corresponding in mobility to purified BSP-A and BSP-C, respectively. Both spots were ninhydrin-positive, stained with dichromate-silver nitrate and. produced alanine (identified by two-dimensional chromatography) after treatment with Raney-nickel. When the synthetic mixture was added to bile, the chromatographic pattern was identical to that of the natural products (Figure 3). Pyrolysis of BSP with glutathione also produced ninhydrin-

$2 \mathrm{By}$ this procedure phenolphthalein also formed complexes with cysteine. Presumably then, the cysteine may conjugate directly with the phenolphthalein core of the BSP molecule. 


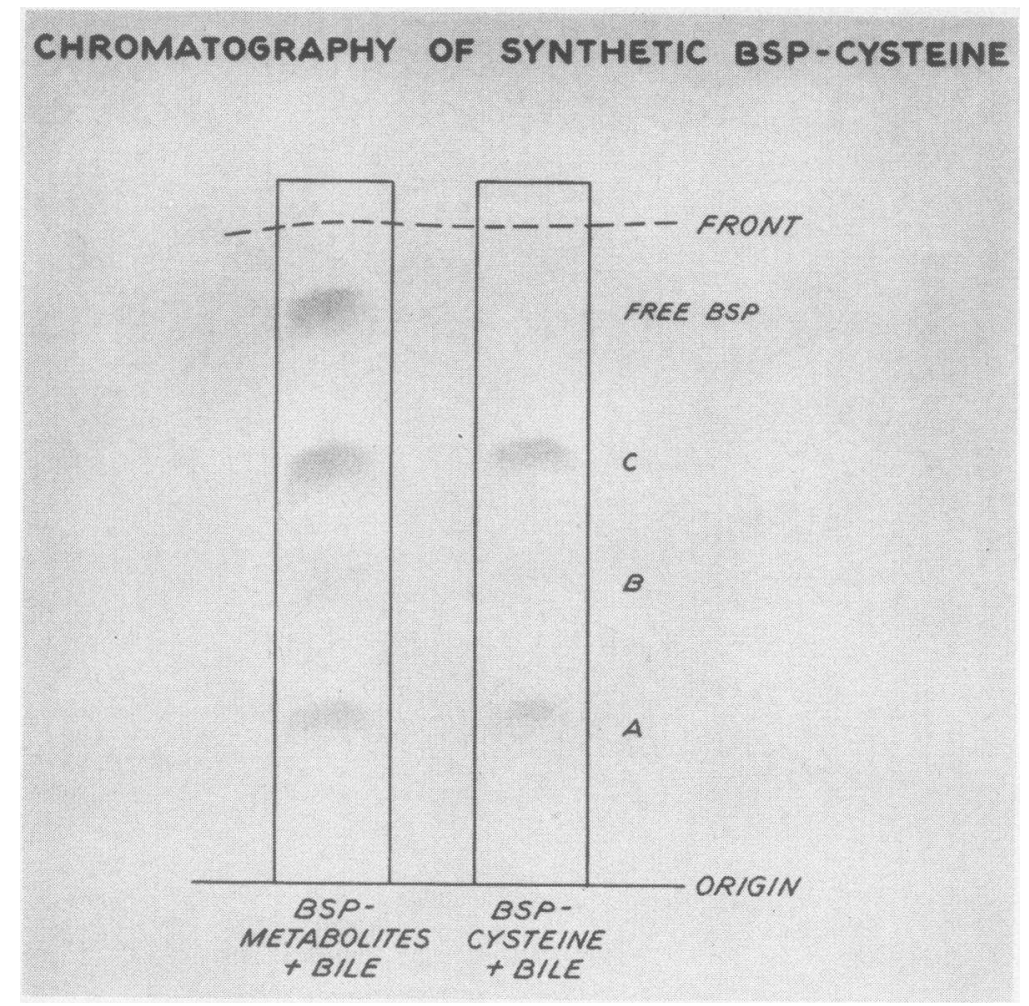

Fig. 3. Chromatography of BSP Metabolites and Synthetic BSPCysteine Complexes

positive synthetic complexes chromatographically similar to BSP-A and BSP-C.

\section{DISCUSSION}

Hydrolysis of the BSP metabolites appearing in human bile with hydrobromic acid, treatment with Raney-nickel, and staining with dichromate-silver nitrate strongly suggest that BSP is excreted, at least in part, as conjugates containing cysteine. Synthetic complexes of BSP and cysteine have the same chromatographic mobilities as the natural metabolites.

The fact that hydrolysis of the metabolites with hydrochloric acid destroys most of the cysteine may explain why the presence of this amino acid was not noted by others $(8,10)$ who used only hydrochloric acid hydrolysis. The appearance of glycine and glutamic acid after hydrolysis of the metabolites may be misleading under circumstances where control bile is not similarly treated (10). Hydrolysis of normal bile purified by our procedures demonstrated that much of the glycine and glutamic acid in the fractions containing the
BSP metabolites may arise from the bile itself or from impurities in the chromatographic paper. However, conclusions based on comparisons of the quantities of amino acids appearing in purified normal bile and in bile containing BSP after acid hydrolysis are also equivocal. The control samples are necessarily taken about 30 minutes before the experimental samples and the two may not be identical in composition. For this reason, the persistent presence of glycine and glutamic acid cannot, at present, be ignored. If glycine and glutamic acid are involved in BSP metabolism, they may be either in combination with the cysteine as a peptide or exist as independent complexes with the same chromatographic mobility as BSP-cysteine mercaptide. In view of the numerous chromatographic systems employed in this study, the latter possibility is unlikely. Furthermore, a conjugation of the acidic BSP molecule with glycine would most likely take place through a peptide linkage, which would result in a ninhydrin-negative compound. The association of cysteine, glycine and glutamic acid with the metabolites 
suggests that glutathione could be involved in BSP metabolism. Although it has never been satisfactorily demonstrated, glutathione has often been suggested as a precursor for cysteine conjugation $(22,23)$. Bray and Franklin showed that liver slices were capable of producing cysteine mercaptides from previously formed glutathione mercaptides (23). We observed that the synthetic BSP-glutathione complexes have chromatographic mobilities similar to those of the naturally occurring metabolites. Isotopic studies, currently under way, may clarify the role of glycine and glutamic acid in the metabolism of BSP.

BSP contains four bromine atoms on one of its three aromatic rings. Precedent for the conjugation of halogenated aromatic substances with cysteine is well known. Two sites for the attachment of cysteine to the BSP molecule are possible: a complex with the $\mathrm{SH}$ group of the cysteine molecule may occur $a$ ) on a free position of an aromatic ring or $b$ ) after substitution for one of the halogens (24). Krebs and Brauer reported that the ratio of bromine to $\mathrm{S}^{35}$ in $\mathrm{BSP}$ and in the metabolites is the same ( 7 ). If so, this observation indicates that no bromine has been lost and that the complexes are at the free positions of the ring. The demonstration that two major complexes can be synthesized from BSP and cysteine suggests two possibilities : that cysteine may complex to different available positions on the BSP molecule or that more than one molecule of cysteine may attach to each BSP molecule. The approximately $1: 1$ molar ratio of $\mathrm{BSP}$ to cysteine in both metabolites favors the former possibility.

The demonstration that BSP is conjugated with cysteine suggests that other tissues may be capable of metabolizing the dye. Mills and Wood (25), utilizing tissue slices, have shown that cysteine conjugation reactions can occur in kidney. Indication of the extrahepatic metabolism of BSP is further suggested by the fact that BSP metabolites account for 25 per cent of the dye excreted in the urine of hepatectomized dogs (26).

After the uptake of circulating BSP by the liver, a significant delay occurs before the pigment is secreted into the bile (27). Apparently at least two processes, both of which may require active enzyme systems, are involved in the clearance of BSP. It is not inconceivable that the conjugation of BSP with cysteine or cysteine-containing pep- tides may be the rate-determining step for either the uptake of the dye by the liver or its eventual secretion into the bile.

\section{SUM MARY}

The two major ninhydrin-positive metabolites of sulfobromophthalein appearing in human bile were isolated by column and paper chromatography. Their chemical characteristics were studied as follows:

1. Hydrolysis of the purified metabolites with concentrated hydrochloric acid at $120^{\circ} \mathrm{C}$. revealed the presence of the amino acids, glycine, glutamic acid and possibly cysteine. Similar treatment of control bile containing no sulfobromophthalein also resulted in the appearance of considerable amounts of glycine and glutamic acid.

2. Hydrolysis of the metabolites with hydrobromic acid more clearly demonstrated the presence of cysteine, which was not detectable in the control bile after similar treatment. Both controls and metabolite fractions contained glycine and glutamic acid.

3. Treatment of both metabolites with Raneynickel resulted in release of desulfurated cysteine (alanine). Controls were negative.

4. Staining with potassium dichromate-silver nitrate gave a positive reaction, indicating the presence of a mercaptide in the metabolites.

5. Synthetic complexes of BSP with cysteine, cystine or glutathione had the same chromatographic mobility as the two naturally occurring metabolites. Amino acids lacking the sulfhydryl group failed to form complexes.

6. It was concluded that BSP is excreted at least in part as a mercaptide with cysteine or the peptide glutathione.

\section{REFERENCES}

1. Brauer, R. W., Krebs, J. S., and Pessotti, R. L. Bromsulfonphthalein as a tool for study of liver physiology (abstract). Fed. Proc. 1950, 9, 259.

2. Carbone, J. V., Grodsky, G. M., and Fanska, R. Evidence for the metabolism of bromsulfalein (BSP). Clin. Res. 1959, 7, 118.

3. Grodsky, G. M., Carbone, J. V., and Fanska, R. Metabolism of sulphobromophthalein. Nature (Lond.) 1959, 183, 469.

4. Brauer, R. W., Pessotti, R. L., and Pizzolato, P. Isolated rat liver preparation. Bile production and other basic properties. Proc. Soc. exp. Biol. (N. Y.) 1951, 78, 174. 
5. Carbone, J. V., Grodsky, G. M., and Hjelte, V. Effect of hepatic dysfunction on circulating levels of sulfobromophthalein and its metabolites. J. clin. Invest. 1959, 38, 1989.

6. Di Somma, A. A. The constitution of conjugated phenolphthalein formed in the animal body. J. biol. Chem. 1940, 133, 277.

7. Krebs, J. S., and Brauer, R. W. Metabolism of sulfobromophthalein sodium (BSP) in the rat. Amer. J. Physiol. 1958, 194, 37.

8. Meltzer, J. I., Wheeler, H. O., and Cranston, W. I. Metabolism of sulfobromophthalein sodium (BSP) in dog and man. Proc. Soc. exp. Biol. (N. Y.) 1959, 100, 175.

9. Monroe, L. S., and Kittinger, A. The metabolism of sulfobromophthalein (BSP) in the human (abstract). Clin. Res. 1959, 7, 119.

10. Combes, B. Biliary excretion by the rat of bromsulfalein as a conjugate of glycine and glutamic acid. Science 1959, 129, 388.

11. Dent, C. E. A study of the behavior of some sixty amino-acids and other ninhydrin-reacting substances on phenol-"collidine" filter-paper chromatograms, with notes as to the occurrence of some of them in biological fluids. Biochem. J. 1948, 43, 169.

12. Block, R. J., and Bolling, I). The Amino Acid Composition of Proteins and Foods, 2nd ed. Springfield, C. C Thomas, 1951, p. 183.

13. Mozingo, R. Catalyst, raney nickel, W-2 in Organic Synthesis, E. C. Horning, Ed. New York, John Wiley and Sons, Inc., 1955, p. 181.

14. Moore, S., and Stein, W. H. Photometric ninhydrin method for use in the chromatography of amino acids. J. biol. Chem. 1948, 176, 367.

15. Durrum, E. L. Two dimensional electrophoresis and ionophoresis. J. Colloid Sci. 1951, 6, 274.

16. Karler, A., Brown, C. L., and Kirk, P. L. A microtechnique for combined analytical or continuous chromatography and electrophoresis. Mikrochim. Acta 1956, p. 1585.

17. Kunkel, H. G. Zone electrophoresis in Methods of Biochemical Analysis, D. Glick, Ed. New York, Interscience Publishers, 1954, vol. 1, p. 141.

18. Schormüller, J., and Leichter, L. Action of various hydrolysis conditions on release of lysine from casein. Z. Lebensmitt.-Untersuch. 1953, 97, 446.

19. Curd, F. H. S., Davis, M. I., Owen, E. C., Rose, F. L., and Tuey, G. A. P. Synthetic antimalarials. VI. Some 4-arylamino-2-aminoalkylamino-6-methylpyrimidines. J. chem. Soc. 1946, 1, 370.

20. Mozingo, R., Wolf, D. E., Harris, S. A., and Folkers, K. Hydrogenolysis of sulfur compounds by Raney Nickel catalyst. J. Amer. chem. Soc. 1943, 65, 1013.

21. Knight, R. H., and Young, I. Biochemical studies of toxic agents. Biochem. J. 1958, 70, 111.

22. Barnes, M. M., and James, S. P. Glutathione and formation of mercapturic acid in a'iz'o. Biochem. J. 1957, 66, 3p.

23. Bray, H. G., and Franklin, T. J. Glutathione and formation of mercapturic acid in aitro. Biochem. J. 1957, 66, 3p.

24. Bray, H. G., James, S. P., and Thorpe, W. V. The metabolism of 3:4-dichloronitrobenzene in the rabbit with special reference to the formation of mercapturic acids. Biochem. J. 1955, 60, xxiii.

25. Mills, G. C., and Wood, J. L. Total mercapturic acid synthesis by liver and kidney. J. biol. Chem. 1954, 207, 695.

26. Rosenau, W., Carbone, J. V., and Grodsky, G. M. Metabolism of sulfobromophthalein in the hepatectomized and hepatectomized-nephrectomized dog. In preparation.

27. Cantarow, A., and Wirts, C. W. Excretion of bromsulfalein in the bile. Proc. Soc. exp. Biol. (N. Y.) 1941, 47, 252. 